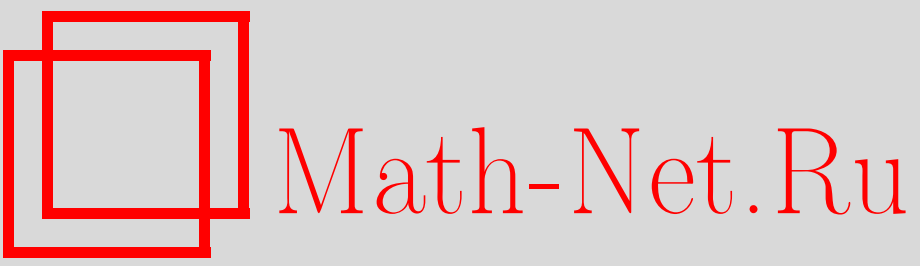

А. Я. Нарманов, А. С. Шарипов, О группе диффеоморфизмов слоеных многообразий, Итоги науки и техн. Сер. Соврем. мат. и ее прил. Темат. обз., 2020, том 181, 74-83

DOI: https://doi.org/10.36535/0233-6723-2020-181-74-83

Использование Общероссийского математического портала Math-Net.Ru подразумевает, что вы прочитали и согласны с пользовательским соглашением

http://www.mathnet.ru/rus/agreement

Параметры загрузки :

IP: 54.80 .97 .219

26 апреля 2023 г., 18:10:21 


\title{
О ГРУППЕ ДИФФЕОМОРФИЗМОВ СЛОЕНЫХ МНОГООБРАЗИЙ
}

\author{
(c) 2020 г. \\ А. Я. НАРМАНОВ, А. С. ШАРИПОВ
}

\begin{abstract}
АннотАция. Целью статьи является введение некоторой топологии на группе $\operatorname{Diff} F(M)$ всех $C^{r}$ диффеоморфизмов слоеного многообразия $(M ; F)$, где $r \geqslant 0$. Эта топология зависит от слоения и называется $F$-компактно-открытой топологией. Она совпадает с компактно-открытой топологией, когда $F$ является $n$-мерным слоением. Если коразмерность слоения равна $n$, то сходимость в этой топологии совпадает с поточечной сходимостью, где $n=\operatorname{dim} M$. Доказано, что некоторые подгруппы группы $\operatorname{Diff}_{F}(M)$ являются топологическими группами с $F$-компактно-открытой топологией. Гладкость всюду в работе означает гладкость класса $C^{\infty}$.
\end{abstract}

Ключевые слова: многообразие, слоение, топологическая группа, компактно-открытая топология.

\section{ON THE DIFFEOMORPHISM GROUPS OF FOLIATED MANIFOLDS}

\author{
(c) 2020 A. YA. NARMANOV, A. S. SHARIPOV
}

\begin{abstract}
In this paper, we introduce a certain topology on the group $\operatorname{Diff}_{F}(M)$ of all $C^{r}$ diffeomorphisms of the foliated manifold $(M ; F)$, where $r \geqslant 0$. This topology depends on the foliation and is called the $F$-compact-open topology. It coincides with the compact-open topology when $F$ is an $n$-dimensional foliation. If the codimension of the foliation is $n$, then the convergence in this topology coincides with the pointwise convergence, where $n=\operatorname{dim} M$. We prove that some subgroups of the group $\operatorname{Diff}_{F}(M)$ are topological groups with the $F$-compact-open topology. Throughout this paper, we use smoothness of the class $C^{\infty}$.
\end{abstract}

Keywords and phrases: manifold, foliation, topological group, compact-open topology.

AMS Subject Classification: 22A05, 54H15, 57R50, 53C12

1. Введение. Множество $\operatorname{Diff}(M)$ всех диффеоморфизмов многообразия на себя является группой относительно композиции и обратного отображения. Группа диффеоморфизмов гладких многообразий имеет важное значение в дифференциальной геометрии и в анализе. Фундаментальными работами в этой области являются исследования В. И. Арнольда, Х. Омори, А. М. Лукацкого (см. [3, 4, 10, 14, 15]).

Интенсивное развитие теории группы диффеоморфизмов началось с работы В. И. Арнольда [10], в которой доказано, что движения идеальной несжимающейся жидкости являются геодезическими на группе диффеоморфизмов, сохраняющих объем элемента.

Когда $M$ является многообразием конечной размерности, то группа изометрий $I(M)$ риманова многообразия $M$ является группой Ли (см. [12]).

Работа выполнена при поддержке Узбекско-Российского гранта фундаментальных исследований (проект MRU № 10-17). 
Х. Омори определил дифференциальную структуру на группе диффеоморфизмов компактных многообразий, которая слабее, чем структура группы Ли в классическом смысле. Эта дифференциальная структура называется структурой ILH (inverse limit of Hilbert) групп Ли. Х. Омори доказал, что ILH-группа Ли диффеоморфизмов может действовать на компактном многообразии транзитивно и неприводимо только в следующих случаях: полная группа диффеоморфизмов, группа диффеоморфизмов, сохраняющих элемент объема, группа симплектических диффеоморфизмов, группа контактных диффеоморфизмов. Вопросы конечной порожденности группы диффеоморфизмов и свойства кривизны ILH-групп Ли диффеоморфизмов исследованы А. М. Лукацким (см. [3, 4]).

В статье рассматриваем слоеное многообразие с некоторым слоением и исследуем некоторые подгруппы группы диффеоморфизмов слоеного многообразия. Известно, что группа диффеоморфизмов является топологической группой в компактно-открытой топологии. В случае, когда многообразие компактно, этот факт доказан в [9]. Для произвольного многообразия конечной размерности этот факт доказан в [13].

2. Основные результаты. Пусть $M$-гладкое связное риманова многообразие размерности $n, 0<k<n$.

Определение 1. Слоением $F$ размерности $k$ (слоением коразмерности $n-k$ ) называется разбиение $M$ на линейно связные подмножества $L_{\alpha} \subset M$, удовлетворяющее следующим свойствам:

$\left(F_{1}\right) \bigcup_{\alpha \in B} L_{\alpha}=M$

$\left(F_{2}\right)$ если $\alpha \neq \beta$, то $L_{\alpha} \cap L_{\beta}=\varnothing$ для всех $\alpha, \beta \in B$;

$\left(F_{3}\right)$ для всякой точки $p \in M$ существуют такие окрестность $U$ и карта $\left(x^{1}, \ldots, x^{k}, y^{1}, \ldots, y^{n-k}\right)$, что для каждого слоя $L_{\alpha}$ компоненты линейной связности множества $U_{p} \cap L_{\alpha}$ задаются уравнениями: $y^{1}=$ const $, \ldots, y^{n-k}=$ const.

Эта карта является отмеченной картой. Компоненты связности множеств $y^{1}=$ const, ..., $y^{n-k}=$ const в отмеченных картах называются локальными слоями слоения $F$. При фиксированном $y$ отображение $x \mapsto(x, y)$ является гладким вложением. Следовательно, локальные слои являются связными $k$-мерными подмногообразиями $M$, каждый слой $L_{\alpha}$ как объединение локальных слоев является $k$-мерным связным подмногообразием $M$. Заметим, что естественная топология на многообразия $L_{\alpha}$ не является топологией, индуцированной из $M$. Простой пример слоения задается гладкой субмерсией $f: M \rightarrow B$, где $B$ является $(n-k)$-мерным многообразием. Компоненты связности полного прообраза точки $y \in B$ определяют $k$-мерное слоение на $M$ (см. [15]).

Через $(M, F)$ обозначим гладкое многообразие $M$ размерности $n$, на котором задано гладкое $k$ мерное слоение $F$ (где $0<k<n)$. Пусть $L(p)$-слой слоения $F$, проходящий через точку $p, T_{p} F-$ касательное пространство слоя $L(p)$ в точке $p$. Имеем подрасслоение (гладкое распределение) $T F=\left\{T_{p} F \mid p \in M\right\}$ касательного расслоения $T M$ многообразия $M$. Обозначим через $V(M)$, $V(F)$ множество гладких сечений расслоений $T M, T F$ соответственно.

Определение 2. Если при диффеоморфизме $f: M \rightarrow M$ образ $f\left(L_{\alpha}\right)$ любого слоя $L_{\alpha}$ слоения $F$ является слоем слоения $F$, то отображение $f: M \rightarrow M$ называется $C^{r}$-диффеоморфизмом слоеного многообразия; этот факт записывается в виде $f:(M, F) \rightarrow(M, F)$.

Пример 1. Пусть $M=\mathbb{R}^{2}(x, y)$ - евклидова плоскость с декартовыми координатами $(x, y)$. Пусть слои $L_{\alpha}$ слоения $F$ задаются уравнениями $x^{2}-y=\alpha=$ const. Тогда диффеоморфизм плоскости $\phi: \mathbb{R}^{2} \rightarrow \mathbb{R}^{2}$, определенный формулой

$$
\phi(x, y)=(x, y+\lambda f(x, y)),
$$

является диффеоморфизмом слоеной плоскости $\left(\mathbb{R}^{2}, F\right)$, для каждого $\lambda \in \mathbb{R}$, удовлетворяющего условию $\lambda \neq 1$, где $f(x, y)=x^{2}-y$. Он отображает каждый слой $L_{\alpha}$ на слой $L_{(1-\lambda) \alpha}$. 
Пример 2. Пусть $(M, F)$ - слоеное многообразие, где $F$ - гладкое слоение размерности $k$, где $0<k<n$. Напомним, что векторное поле $X$ называется слоеным полем, если для каждого векторного поля $Y$, касательного к слоению $F$, скобка Ли $[X, Y]$ также является касательным к $F$. Известно, что поток каждого слоеного поля состоит из диффеоморфизмов слоеного многообразия $(M, F)$. Для слоеной плоскости из примера 1 векторное поле $X=\left(x^{2}-y\right) \frac{\partial}{\partial y}$ является слоеным полем,
и его поток состоит из диффеоморфизмов

$$
\phi^{t}:(x, y) \in \mathbb{R}^{2} \rightarrow\left(x, x^{2}-e^{-t}\left(x^{2}-y\right)\right) \in \mathbb{R}^{2}
$$

слоеной плоскости $\left(\mathbb{R}^{2}, F\right)$. Каждый диффеоморфизм (1) отображает каждый слой $L_{\alpha}$ на слой $L_{e^{-t} \alpha}$.

Следующий пример показывает, что понятие диффеоморфизм слоеного многообразия определено корректно.

Пример 3. Пусть $M=\mathbb{R}^{2}(x, y)$ - евклидова плоскость с декартовыми координатами $(x, y)$. Слои $L_{\alpha}$ слоения $F$ задаются уравнениями $y=\alpha=$ const. Тогда гомеоморфизм плоскости $\phi: \mathbb{R}^{2} \rightarrow \mathbb{R}^{2}$, определенный формулой

$$
\phi(x, y)=\left(x+y, y^{1 / 3}\right)
$$

является диффеоморфизмов на каждом слое слоения $F$, но не является диффеоморфизмом плоскости.

Обозначим через $\operatorname{Diff}_{F}(M)$ множество всех $C^{r}$-диффеоморфизмов слоеного многообразия $(M, F)$, где $r \geqslant 0$. Множество $\operatorname{Diff}^{r}(M)$ является группой по отношению к суперпозиции и обратного отображения. Группа $\operatorname{Diff}_{F}(M)$ является подгруппой $\operatorname{Diff}(M)$; она является топологической группой в компактно-открытой топологии.

Введем некоторую топологию на группе $\operatorname{Diff}_{F}(M)$, которая зависит от слоения $F$ и совпадает с компактно-открытой топологией, когда $F$ является $n$-мерным слоением.

Пусть $\left\{K_{\lambda}\right\}$ - семейство всех компактных множеств, где каждое $K_{\lambda}$ принадлежит какомулибо слою слоения $F$, и пусть $\left\{U_{\beta}\right\}$ - семейство всех открытых множеств на $M$. Рассмотрим для каждой пары $K_{\lambda} \subset L_{\alpha}$ и любого $U_{\beta}$ совокупность всех отображений $f \in \operatorname{Diff}_{F}(M)$, для которых $f\left(K_{\lambda}\right) \subset U_{\beta}$. Эту совокупность отображений будем обозначать через

$$
\left[K_{\lambda}, U_{\beta}\right]=\left\{f: M \rightarrow M \mid f\left(K_{\lambda}\right) \subset U_{\beta}\right\} .
$$

Нетрудно показать, что семейство всевозможных конечных пересечений множеств вида $\left[K_{\lambda}, U_{\beta}\right]$ образует базу для некоторой топологии. Эту топологию назовем слоеной компактно-открытой топологией или коротко $F$-компактно-открытой топологией.

Пусть $M$ - гладкое связное риманово многообразие конечной размерности.

Определение 3. Изометрия $\phi: M \rightarrow M$ называется изометрией слоеного многообразия $(M, F)$, если она переводит слой на слой, т.е. является диффеоморфизмом слоеного многообразия $(M, F)$.

Пример 4. Пусть $M=\mathbb{R}^{2}(x, y)$ - евклидова плоскость с декартовыми координатами $(x, y)$. Пусть слоение $F$ задается уравнением $x^{2}-y=\alpha=$ const. Тогда изометрия плоскости $\phi: \mathbb{R}^{2} \rightarrow \mathbb{R}^{2}$, определенная формулой

$$
\phi_{\lambda}(x, y)=(x, y+\lambda)
$$

является изометрией слоеной плоскости $\left(\mathbb{R}^{2}, F\right)$ для каждого $\lambda \in \mathbb{R}$. Она отображает каждый слой $L_{\alpha}$ на слой $L_{\alpha}-\lambda$. Семейство изометрий $\phi_{\lambda}$ является потоком векторного поля Киллинга $X=\partial / \partial y$. Нетрудно проверить, что диффеоморфизм слоеной плоскости из примера 1 является изометрией слоеной плоскости, но не является потоком векторного поля.

Напомним, что векторное поле $X$ на римановом многообразии $(M, g)$ называется векторным полем Киллинга, если поток состоит из изометрий риманова многообразия $(M, g)$, т.е. $L_{X} g=0$, где риманова метрика, а через $L_{X} g$ обозначена производная Ли метрики по направлению $X$. 
Геометрия орбит семейства векторных полей Киллинга изучена в [6]. Если $X$ является слоеным векторным полем Киллинга, то его поток состоит из изометрий слоеного многообразия $(M, F)$.

Обозначим через $\operatorname{Iso}_{F}(M)$ множество всех $C^{r}$-изометрий слоеного многообразия $(M, F)$, где $r \geqslant 0$. Имеет место $\operatorname{Iso}_{F}(M)=\operatorname{Diff}_{F}(M) \cap \operatorname{Iso}(M)$.

Теорема 1. Пусть $(M, F)$ - гладкое связное полное слоеное риманово многообразие конечной размерности. Тогда группа $\operatorname{Iso}_{F}(M)$ является топологической группой с $F$-компактно-открытой топологией.

Доказательство. Сначала покажем, что отображение $(g, h) \rightarrow g \circ h$ является непрерывным для отображений $g, h \in \operatorname{Iso}_{F}(M)$. Пусть $g \circ h \in[K, G]$, где $K$ - компактное подмножество некоторого слоя $L$ слоения $F, G$-открытое подмножество многообразия $M$. Отсюда имеем $h(K) \subset g^{-1}(G)$. Так как $h(K)$ является компактным множеством, существует открытое покрытие, состоящее из конечного числа открытых подмножеств $U_{1}, \ldots, U_{m}$ слоя $h(L)$ с компактным замыканием в $h(L)$, содержащихся в $g^{-1}(G)$. (Так как слой $h(L)$ локально компактен и хаусдорфов, каждая точка $h(K)$ обладает окрестностью в $h(K)$ с компактным замыканием в $h(L)$, лежащим в $g^{-1}(G)$.)

Положим $A=\bigcup_{i=1}^{m} U_{i}$. Тогда $g \in[\bar{A}, G], h \in\left[K, A^{\prime}\right]$, где $A^{\prime}$ - такое открытое подмножество множества $M$, что $A \subset A^{\prime} \subset g^{-1}(G)$. Отсюда следует, что $(g, h) \in[\bar{A}, G] \times\left[K, A^{\prime}\right]$, когда $g \circ h \in[K, G]$.

Теперь обозначим через $d(x, y)$ расстояние между точками $x$ и $y$, определяемое полной римановой метрикой $g$. Используя полноту римановой метрики на $M$, докажем, что отображение $\chi: f \rightarrow f^{-1}$ непрерывно. Для этого докажем, что прообраз $\chi^{-1}(A)$ открытого множества $A \subset \operatorname{Iso}_{F}(M)$ является открытым. В самом деле, достаточно доказать этот факт, когда $A$ является элементом предбазы, т.е. $A$ имеет вид

$$
A=\left\{f \in \operatorname{Iso}_{F}(M): f(K) \subset V\right\}
$$

где $K$-компакт, $V$ - открытое множество. В этом случае прообраз множества $A$ имеет вид

$$
\chi^{-1}(A)=\left\{f \in \operatorname{Iso}_{F}(M): f^{-1}(K) \subset V\right\} .
$$

Покажем, что $\chi^{-1}(A)$ открыто в $F$-компактно-открытой топологии.

Пусть $g \in \chi^{-1}(A), U$ - такая окрестность $g^{-1}(K)$ с компактным замыканием, что $\bar{U} \subset V$.

Положим

где

$$
K_{1}=g^{-1}(K), \quad U_{g}=\left\{h \in \operatorname{Iso}_{F}(M): d(g(x), h(x))<\frac{\varepsilon}{2} \forall x \in K_{1}\right\},
$$

$$
\varepsilon=d\left(K_{1}, M \backslash U\right)=\inf \left\{d(x, y): x \in K_{1}, y \in(M \backslash U)\right\} .
$$

Множество $U_{g}$ является открытым множеством в $F$-компактно-открытой топологии в силу того, что $U_{g}=\left[K_{1}, V_{\varepsilon}\right]$, где $V_{\varepsilon}=\{x \in M: d(x, K)<\varepsilon\}$ - окрестность $K, d(x, K)=\inf \{d(x, y): y \in K\}-$ расстояние от точки $x$ до множества $K$.

Покажем, что если $h \in U_{g}$, то $h^{-1}(K) \subset V$, т.е. $U_{g} \subset \chi^{-1}(A)$. Предположим обратное. Пусть для некоторого $h \in U_{g}$ существует такая точка $y \in K$, что $h^{-1}(y) \in M \backslash U$, т.е. $y \in M \backslash h(U)$. Тогда в силу того, что $g^{-1}(y) \in K_{1}$, имеет место неравенство

$$
d\left(y=g\left(g^{-1}(y)\right), h\left(g^{-1}(y)\right)\right)<\frac{\varepsilon}{2} .
$$

Пусть $\gamma$ - кратчайшая (известно, что на полном римановом многообразии для каждой пары точек всегда существует кратчайшая геодезическая, которая соединяет их), идущая из точки $у$ в точку $h\left(g^{-1}(y)\right)$, а $z \in \gamma \cap \partial(h(U))$. Тогда

$$
d\left(h\left(g^{-1}(y)\right), z\right)<\frac{\varepsilon}{2} .
$$

Учитывая, что $h^{-1}$ - изометрия, имеем

$$
d\left(h\left(g^{-1}(y)\right), z\right)=d\left(g^{-1}(y), h^{-1}(z)\right) .
$$


$\mathrm{C}$ другой стороны, имеем $g^{-1}(y) \in K_{1}, h^{-1}(z) \in \partial U$ и $d\left(g^{-1}(y), h^{-1}(z)\right) \geqslant \varepsilon$. Это противоречие показывает, что $h^{-1}(K) \subset U$. Следовательно, $U_{g} \subset \chi^{-1}(A)$.

Пусть $M$ - гладкое полное риманово многообразие размерности $n$ с гладким слоением размерности $k$, где $0<k<n$. Тогда каждый слой является римановым многообразием с индуцированной римановой метрикой. Следующая теорема показывает, что если $M$ является полным римановым многообразием, то каждый слой является полным римановым многообразием с индуцированной римановой метрикой.

Теорема 2. Пусть $M$ - гладкое полное риманово многообразие размерности п с гладким слоением размерности $k$, где $0<k<n$. Тогда справедливы следующие утверждения.

1. Каждый слой с индуцированной римановой метрикой на нем является полным римановым многообразием.

2. Пусть $\gamma_{m}: \mathbb{R}^{1} \rightarrow L_{m}$ - такал последовательность геодезических (относительно индуцированных римановых метрик) слоев $L_{m}$, что $\gamma_{m}\left(s_{0}\right) \rightarrow p$ nри $m \rightarrow \infty$ для некоторого $s_{0} \in \mathbb{R}^{1}$. Тогда существует подпоследовательность $\gamma_{m_{l}}$ последовательности $\gamma_{m}$, которая поточечно сходится к некоторой геодезической $\gamma: \mathbb{R}^{1} \rightarrow L(p)$ слоя $L(p)$, выходящей из точки $p$ при $s=s_{0}$.

Доказательство. 1. Пусть $L_{\alpha}-$ некоторый слой слоения $F, \gamma:(a, b) \rightarrow L_{\alpha}$ - геодезическая на $L_{\alpha}$, определенной индуцированной римановой метрикой и параметризованная длиной дуги. Покажем, что если $b<\infty$ (или $a>-\infty$ ), то существует $\lim _{s \rightarrow b} \gamma(s)$ или $\lim _{s \rightarrow a} \gamma(s)$, принадлежащий $L_{\alpha}$. Пусть $s_{1}<s_{2}<\cdots<s_{l}<s_{l+1}<\ldots, s_{l} \rightarrow b$ при $l \rightarrow \infty$, и $\varepsilon>0$ - некоторое малое число. Тогда существует такой номер $N$, что

$$
d\left(\gamma\left(s_{i}\right), \gamma\left(s_{j}\right)\right) \leqslant\left|s_{i}-s_{j}\right|<\varepsilon \quad x \text { для всех } i, j \geqslant N,
$$

где $d$ - расстояние на $M$. Следовательно, последовательность $\left\{\gamma\left(s_{l}\right)\right\}$ является фундаментальной. В силу того, что $M$ полно, эта последовательность имеет предел, который мы обозначим через $p$. Теперь покажем, что $p \in L_{\alpha}$. По определению слоения, для каждой точки $p \in M$ существуют такие окрестность $U$ точки $p$ и локальная система координат $\left(x^{1}, \ldots, x^{k}, y^{1}, \ldots, y^{n-k}\right)$ на $U$, что набор $\left\{\frac{\partial}{\partial x^{1}}, \ldots, \frac{\partial}{\partial x^{k}}\right\}$ является базисом для гладких сечений $\left.T F\right|_{U}$ (сужение $T F$ на $U$ ). Такая окрестность называется расслоенной окрестностью точки $p$. Пусть $U$ - расслоенная окрестность точки $p$ с локальными координатами $\left(x^{1}, \ldots, x^{k}, y^{1}, \ldots, y^{n-k}\right)$. Тогда связные компоненты пересечения $V \cap L_{\beta}$ для любого слоя $L_{\beta}$ описываются уравнениями $y^{1}=$ const, $\ldots, y^{n-k}=$ const.

Если $L_{0}$ - компонента связности пересечения $V \cap L_{\alpha}$, содержащая точки $\gamma\left(s_{i}\right)$ для достаточно больших $i$, то ясно, что $L_{0}$ содержит точку $p$. Следовательно, $p \in L_{\alpha}$. Отсюда следует, что $\gamma(s)$ определено для всех $s \in(-\infty,+\infty)$.

2. Пусть $\pi: T M \rightarrow T F$-ортогональная проекция, $V(M), V(F), V(H)$-множество гладких сечений расслоений $T M, T F, H F$ соответственно. Положим $\widetilde{\nabla}_{X} Y=\pi\left(\nabla_{X} Y\right)$ для векторных полей $X \in V(M), Y \in V(F)$, где $\nabla$ - связность Леви-Чивиты, определенная римановой метрикой $g$ на $M$. Известно, что $\widetilde{\nabla}_{X} Y$ является связностью на $T F$, причем ее сужение на каждый слой $L_{\alpha}$ совпадает со связностью на $L_{\alpha}$, определяемой индуцированной римановой метрикой на $L_{\alpha}$ из $M$ (см. $[2,5])$. Поэтому гладкая параметризованная кривая $\mu:(a, b) \rightarrow M$, лежащая на слое $L_{\alpha}$ слоения $F$, является геодезической на $L_{\alpha}$ (относительно индуцированной римановой метрики) тогда и только тогда, когда

$$
\widetilde{\nabla}_{\dot{\mu}} \dot{\mu}=0 \text {. }
$$

Если $\mu$ лежит в отмеченной окрестности $U$, то ее уравнения имеют вид:

$$
x^{i}=x^{i}(s), \quad y^{\alpha}=\text { const }, \quad 1 \leqslant i \leqslant k, \quad 1 \leqslant \alpha \leqslant n-k .
$$

Так как для $\nabla$ имеет место равенство

$$
\nabla_{\frac{\partial}{\partial x^{i}}} \frac{\partial}{\partial x^{j}}=\Gamma_{i, j}^{l} \frac{\partial}{\partial x^{l}}+\Gamma_{i, j}^{\alpha} \frac{\partial}{\partial y^{\alpha}},
$$


то имеем

$$
\widetilde{\nabla}_{\frac{\partial}{\partial x^{i}}}=\Gamma_{i, j}^{l} \frac{\partial}{\partial x^{l}},
$$

где $1 \leqslant i, j, l \leqslant k, 1 \leqslant \alpha \leqslant n-k, \Gamma_{i, j}^{\beta}$ - символы Кристоффеля. Отсюда и из свойств оператора $\widetilde{\nabla}$ вытекает, что уравнение (2) эквивалентно следующей системе дифференциальных уравнений второго порядка:

$$
\frac{d^{2} x^{i}}{d s}+\Gamma_{l, j}^{i} \frac{d x^{l}}{d s} \frac{d x^{j}}{d s}=0
$$

Полагая $u^{i}=d x^{i} / d s$, эту систему можно написать в виде

$$
\frac{d x^{i}}{d s}=u^{i}, \quad \frac{d u^{i}}{d s}=-\Gamma_{l, j}^{i} u^{l} u^{j}
$$

Не ограничивая общности, будем считать, что геодезические $\gamma_{m}: \mathbb{R}^{1} \rightarrow L_{m}$ параметризованы длиной дуги и $s_{0}=0$. Покажем, что из последовательности касательных векторов $\dot{\gamma}_{m}(0)$ можно выделить сходящую подпоследовательность. Так как $\left|\dot{\gamma}_{m}(0)\right|=1$ для всех $m$, имеем равенство

$$
\sum_{i, j=1}^{k} g_{i j}\left(p_{m}\right) u_{m}^{i} u_{m}^{j}=1
$$

для достаточно больших $m$, где $p_{m}=\gamma_{m}(0), g_{i j}$ - коэффициент римановой метрики $g,\left\{u_{m}^{i}\right\}-$ первые $k$ координат вектора $\dot{\gamma}_{m}(0)$ в координатах расслоенной окрестности $U$ точки $p$. Равенство (4) имеет смысл при $\gamma_{m}(0) \in U$. Так как $p_{m} \rightarrow p$ при $m \rightarrow \infty$, для любого $\varepsilon>0$ существует такое $m_{0}$, для которого $\left|g_{i j}\left(p_{m}\right)-g_{i j}(p)\right|<\varepsilon$ при $m \geqslant m_{0}$. Отсюда имеем

$$
\sum_{i, j=1}^{k}\left(g_{i j}(p)-\varepsilon\right)\left|u_{m}^{i}\right|\left|u_{m}^{j}\right| \leqslant \sum_{i, j=1}^{k} g_{i j}\left(p_{m}\right)\left|u_{m}^{i}\right|\left|u_{m}^{j}\right| .
$$

В силу того, что матрица $\left\{g_{i j}\left(p_{m}\right)\right\}$ положительно определена, каждое уравнение

$$
\sum_{i, j=1}^{k} g_{i j}\left(p_{m}\right) u^{i} u^{j}=1
$$

определяет эллипсоид, симметричный относительно осей координат и с центром симметрии в начале координат. Поэтому имеем

следовательно,

$$
\sum_{i, j=1}^{k} g_{i j}\left(p_{m}\right)\left|u_{m}^{i}\right|\left|u_{m}^{j}\right|=1
$$

$$
\sum_{i, j=1}^{k}\left(g_{i j}(p)-\varepsilon\right)\left|u_{m}^{i}\right|\left|u_{m}^{j}\right| \leqslant 1 .
$$

При достаточно малом $\varepsilon>0$, матрица $\left\{\left(g_{i j}(p)-\varepsilon\right)\right\}$ также является положительно определенной. Отсюда следует, что точки $u_{m}=\left\{u_{m}^{1}, \ldots, u_{m}^{k}\right\}$ лежат на компактном множестве. Поэтому из последовательности $u_{m}^{i}$ можно выделить сходящуюся подпоследовательность $u_{m_{l}}^{i}$, предел которой обозначим через $u_{0}^{i}$. Тогда для вектора $v=\left(u_{0}^{1}, \ldots, u_{0}^{k}\right)$ имеет место соотношение

$$
\sum_{i, j=1}^{k} g_{i j}(p)\left|u_{0}^{i}\right|\left|u_{0}^{j}\right|=1 .
$$

Рассмотрим геодезическую $\gamma$ на слое $L(p)$, выходящую из точки $p$ при $s=0$ в направлении вектора $v$. Эта кривая удовлетворяет уравнению (3). Пусть $K_{0} \subset \mathbb{R}^{1}$ - такой компакт, содержащий $s_{0}=0$, что $\gamma\left(K_{0}\right)$ лежит в $U$. Тогда $\gamma: K_{0} \rightarrow L(p)$ является решением системы дифференциальных уравнений (3) с начальными условиями

$$
x^{i}(0)=p^{i}, \quad u^{i}(0)=v^{i}
$$


где $i=1, \ldots, k, p=\left(p^{1}, \ldots, p^{n}\right), v=\left(u_{0}^{1}, \ldots, u_{0}^{n}\right)$.

Так как $\gamma_{m_{l}}(0) \rightarrow p, \dot{\gamma}_{m_{l}}(0) \rightarrow v$ при $l \rightarrow \infty$, то по теореме о непрерывной зависимости решения дифференциальных уравнений от начальных данных последовательность $\gamma_{m}$ сходится к $\gamma$ равномерно на компакте $K_{0} \subset(a, b)$. Для каждого компакта $K \subset \mathbb{R}^{1}$, содержащего $K_{0}$, покрывая $\gamma(K)$ расслоенными окрестностями, получим, что $\gamma_{m_{l}}$ сходится к $\gamma$ равномерно на $K$.

Обозначим через $\operatorname{Diff}_{F}^{0}(M)$ множество всех таких $C^{r}$-диффеоморфизмов $g \in \operatorname{Diff}_{F}(M)$ слоеного многообразия $(M, F)$, что $g\left(L_{\alpha}\right)=L_{\alpha}$ для каждого слоя $L_{\alpha}$ слоения $F$. Поток каждого касательного векторного поля состоит из диффеоморфизмов слоеного многообразия $(M, F)$, которые принадлежат группе $\operatorname{Diff}_{F}^{0}(M)$. Можно доказать следующую теорему.

Теорема 3. Пусть $(M, F)$ - слоеное многообразие, где $M$ - гладкое связное многообразие конечной размерности. Тогда группа $\operatorname{Diff}_{F}^{0}(M)$ является топологической группой с $F$-компактнооткрытой топологией.

Доказательство. Непрерывность отображения $(g, h) \rightarrow g \circ h$ доказана в теореме 1. Обозначим через $d(x, y)$ расстояние между точками $x$ и $y$, определяемое некоторой полной римановой метрикой. Известно, что гладкое многообразие $M$ обладает полной римановой метрикой (см. [1]).

Пусть $\pi: T M \rightarrow T F-$ ортогональная проекция. Предположим, что $\tilde{\nabla}_{X} Y=\pi\left(\nabla_{X} Y\right)$ для векторных полей $X \in V(M), Y \in V(F)$, где $\nabla$ - связность Леви-Чивиты, определенная римановой метрикой $g$ на $M$. Известно, что $\tilde{\nabla}_{X} Y$ является связностью на $T F$, причем ее сужение на каждый слой $L_{\alpha}$ совпадает со связностью на $L_{\alpha}$, определяемой римановой метрикой на $L_{\alpha}$ из $M$ (см. [2,3]).

Докажем, что отображение $\chi: f \rightarrow f^{-1}$ непрерывно. Пусть $g$ - полная риманова метрика на $M$. Сужение римановой метрики $g$ индуцирует риманову метрику на слоях. Эта индуцированная риманова метрика определяет функцию расстояния на каждом слое.

Пусть $L_{\alpha}$ - некоторый слой слоения $F, d_{\alpha}$ - расстояние на $L_{\alpha}$, определенное индуцированной римановой метрикой $g_{\alpha}$. По теореме 2 каждый слой $L_{\alpha}$ индуцированной римановой метрики является полным римановым многообразием.

Теперь, используя полноту римановой метрики $d_{\alpha}$, докажем непрерывность отображения $\chi: f \rightarrow f^{-1}$. Для этого проверим, что полный прообраз $\chi^{-1}(A)$ открытого множества $A \subset \operatorname{Diff}_{F}^{0}(M)$ является открытым. В самом деле, достаточно показать этот факт, когда $A$ является элементом предбазы, т.е. $A$ имеет вид

$$
A=\left\{f \in \operatorname{Diff}_{F}^{0}(M): f(K) \subset V\right\}
$$

где $K$ - компактное подмножество слоя $L_{\alpha}, V$ - открытое подмножество многообразия $M$. В этом случае прообраз множества $A$ имеет вид

$$
\chi^{-1}(A)=\left\{f \in \operatorname{Diff}_{F}^{0}(M): f^{-1}(K) \subset V\right\} .
$$

Покажем, что $\chi^{-1}(A)$ открыто в $F$-компактно-открытой топологии. Пусть $g \in \chi^{-1}(A), U$ - такая окрестность $g^{-1}(K)$ в $L_{\alpha}$, с компактным замыканием $\bar{U}$ в $L_{\alpha}$, что $\bar{U} \subset V$. Положим

где

$$
U(g)=\left\{h \in \operatorname{Diff}_{F}^{0}(M): d_{\alpha}(g(x), h(x))<\frac{\varepsilon}{2} \forall x \in \bar{U}\right\},
$$

$$
\varepsilon=d\left(K, L_{\alpha} \backslash g(U)\right)=\inf \left\{d_{\alpha}(x, y): x \in K, y \in L_{\alpha} \backslash g(U)\right\} .
$$

Покажем, что если $h \in U(g)$, то $h^{-1}(K) \subset V$, т.е. $U(g) \subset \chi^{-1}(A)$. Предположим обратное. Пусть для некоторого $h \in U(g)$ существует такая точка $y \in K$, что $h^{-1}(y) \in L_{\alpha} \backslash U$, т.е. $y \in L_{\alpha} \backslash h(U)$. Тогда в силу того, что $g^{-1}(y) \in U$, имеет место неравенство

$$
d\left(y=g\left(g^{-1}(y)\right), h\left(g^{-1}(y)\right)\right)<\frac{\varepsilon}{2} .
$$

Пусть $\gamma$ - кратчайшая на $L_{\alpha}$ (так как $\left(L_{\alpha}, d_{\alpha}\right)$ - многообразие полное риманово, для каждой пары точек всегда существует кратчайшая геодезическая, которая соединяет их), идущая из точки $y$ в точку $h\left(g^{-1}(y)\right)$, а $z \in \gamma \cap \partial(h(U))$. Тогда $h^{-1}(z) \in \bar{U}$ и, кроме того,

$$
d\left(g\left(h^{-1}(z)\right), h\left(h^{-1}(z)\right)\right)<\frac{\varepsilon}{2} .
$$


С другой стороны, $d(y, z)<\varepsilon / 2$. Следовательно,

$$
d\left(y, g\left(h^{-1}(z)\right)\right) \leqslant d(y, z)+d\left(z, g\left(h^{-1}(z)\right)\right)<\varepsilon .
$$

Но, с другой стороны, $z \notin h(U)$; кроме того, имеем

$$
g\left(h^{-1}(z)\right) \in L_{\alpha} \backslash g(U), \quad d b\left(y, g\left(h^{-1}(z)\right)\right) \geqslant \varepsilon .
$$

Полученное противоречие показывает, что $h^{-1}(K) \subset U$. Следовательно, $U(g) \subset \chi^{-1}(A)$.

Теперь рассмотрим еще одну подгруппу группы $\operatorname{Diff}_{F}(M)$. Обозначим через $G_{F}(M)$ множество всех $g \in \operatorname{Diff}_{F}(M)$, которые являются изометрией на каждом слое слоения $F$, т.е. для каждого слоя $L_{\alpha}$ слоения $F$ сужение $g: L_{\alpha} \rightarrow g\left(L_{\alpha}\right)$ является изометрией.

Замечание 1. Если $r \geqslant 1$, то для каждого элемента $\varphi \in G_{F}(M)$ дифференциал $d \varphi$ сохраняет длину каждого касательного вектора $v \in T_{p} F$, т.е. имеет место $\left|d \varphi_{p}(v)\right|=|v|$ при любом $p$. Если же $r=0$, то каждый элемент $\varphi$ из $G_{F}(M)$ является гомеоморфизмом многообразия $M$. Риманова метрика многообразия $M$ индуцирует на каждом слое $L_{\alpha}$ риманову метрику, которая определяет на нем расстояние. В этом случае $\varphi$ является изометрией между метрическими пространствами $L_{\alpha}$ и $\varphi\left(L_{\alpha}\right)$. Тогда по известной теореме $\varphi$ является диффеоморфизмом $L_{\alpha}$ на $\varphi\left(L_{\alpha}\right)$ для каждого слоя $L_{\alpha}$ (см. [11]).

Теперь докажем вспомогательные леммы.

Лемма 1. Предположим, что $\left\{f_{m}\right\} \in G_{F}(M)$ - последовательность, которая поточечно сходится на множестве $A \subset L_{\alpha}$, где $L_{\alpha}-$ некоторый слой слоения $F$. Тогда $\left\{f_{m}\right\}$ также поточечно сходится на $\bar{A}$ (әде $\bar{A}-$ замыкание множества $A$ в $\left.L_{\alpha}\right)$.

Доказательство. Пусть $p \in \bar{A}, \varepsilon>0$. Выберем сначала такую точку $p_{1} \in A$, что $d_{\alpha}\left(p, p_{1}\right)<\varepsilon / 3$, и такое $N$, что

$$
d\left(f_{l}\left(p_{1}\right), f_{m}\left(p_{1}\right)\right)<\frac{\varepsilon}{3}, \quad l, m \geqslant N,
$$

где $d_{\alpha}\left(p, p_{1}\right)$ - расстояние между точками $p$ и $p_{1}$ на слое $L_{\alpha}$. Тогда

$$
d\left(f_{l}(p), f_{m}(p)\right) \leqslant d\left(f_{l}(p), f_{l}\left(p_{1}\right)\right)+d\left(f_{l}\left(p_{1}\right), f_{m}\left(p_{1}\right)\right)+d\left(f_{m}\left(p_{1}\right), f_{m}(p)\right)<\varepsilon
$$

для всех $m, n \geqslant N$. Следовательно, последовательность $\left\{f_{m}(p)\right\}$ фундаментальна, и из полноты многообразия $M$ получаем, что $\left\{f_{m}(p)\right\}$ сходится.

Лемма 2. Пусть $A$-такое множество точек на слое $L_{\alpha}$, что для каждой точки $p \in A$ существует сходящаяся подпоследовательность $f_{m_{l}}(p)$ последовательности $f_{m}(p)$. Если множество $A$ непусто, то $A=L_{\alpha}$.

Доказательство. Пусть $p \in L_{\alpha}, p^{*} \in A, r=d_{\alpha}\left(p, p^{*}\right)$, где $d_{\alpha}\left(p, p^{*}\right)$ - расстояние между точками $p$ и $p^{*}$ на $L_{\alpha}$. Пусть подпоследовательность $\left\{f_{m_{l}}\right\}$ такова, что $\left\{f_{m_{l}}\left(p^{*}\right)\right\}$ сходится. Так как $f_{m_{l}}-$ изометрия слоения, то расстояние $d_{\alpha}\left(p, p^{*}\right)$ между точками $p$ и $p^{*}$ на слое $L_{\alpha}$ сохраняется и

$$
d_{f_{l}(\alpha)}\left(f_{m_{l}}(p), f_{m_{l}}\left(p^{*}\right) b\right)=d_{\alpha}\left(p, p^{*}\right),
$$

где $d_{f_{l}(\alpha)}$ - расстояние на слое $f_{m_{l}}\left(L_{\alpha}\right)$. Пусть $q^{*}=\lim _{l \rightarrow \infty}\left(f_{m_{l}}\left(p^{*}\right)\right)$. Тогда

$$
d\left(q^{*}, f_{m_{l}}(p)\right) \leqslant d\left(q^{*}, f_{m_{l}}\left(p^{*}\right)\right)+d\left(f_{m_{l}}\left(p^{*}\right), f_{m_{l}}(p)\right) \leqslant \varepsilon+r .
$$

Следовательно, множество $\left\{f_{m_{l}}(p)\right\}$ имеет компактное замыкание. Отсюда следует, что $p \in A$.

Следующая теорема показывает некоторое свойство группы $G_{F}(M)$ с $F$-компактно-открытой топологией.

Теорема 4. Пусть $M$ - полное гладкое многообразие размерности $n$ с гладким слоением $F$ размерности $k, f_{m} \in G_{F}^{r}(M), r \geqslant 0, m=1,2, \ldots$ Предположим, что для каждого слоя $L_{\alpha}$ существует такая точка $o_{\alpha} \in L_{\alpha}$, что последовательность $f_{m}\left(o_{\alpha}\right)$ сходится. Тогда существует подпоследовательность $f_{m_{l}}$ последовательности $f_{m}$, сходящаяся в $F$-компактно-открытой топологии. 
Доказательство. Пусть $L_{\alpha}-$ произвольный слой. По условиям теоремы существует такая точка $o_{\alpha} \in L_{\alpha}$, что последовательность $\left\{f_{m}\left(o_{\alpha}\right)\right\}$ сходится. По лемме 2 для каждой точки $p \in L_{\alpha}$ последовательность $\left\{f_{m}(p)\right\}$ содержит сходящуюся подпоследовательность. Отсюда следует, что последовательность $\left\{f_{m}(p)\right\}$ содержит сходящуюся подпоследовательность для всех $p \in M$.

Риманово многообразие $M$ является сепарабельным метрическим пространством. Поэтому оно содержит всюду плотное счетное подмножество $A=\left\{p_{i}\right\}$. Для каждой точки $p_{i}$ существует сходящаяся подпоследовательность $\left\{f_{m_{l}}^{i}\left(p_{i}\right)\right\}$ последовательности $\left\{f_{m}\left(p_{i}\right)\right\}$. Используя диагональный процесс, можем найти подпоследовательность $\left\{f_{m_{l}}\right\}$, которая сходится во всех точках множества $A$. Так как для каждого слоя $L_{\alpha}$ существует точка $o_{\alpha} \in L_{\alpha}$, в которой последовательность $\left\{f_{m_{l}}\right\}$ сходится, то по лемме 2 она сходится во всех точках слоя $L_{\alpha}$. Отсюда получим, что последовательность сходится поточечно в каждой точке $M$.

Теперь, полагая $\varphi(p)=\lim _{l \rightarrow \infty} f_{m_{l}}$, получим отображение $\varphi: M \rightarrow M$. Пусть $p \in L_{\alpha}$ для некоторого слоя $L_{\alpha}, \gamma:[0, l] \rightarrow L_{\alpha}$ - геодезическая, которая реализует расстояние $d_{0}=d_{\alpha}\left(o_{\alpha}, p\right)$ на слое $L_{\alpha}$, и параметризованная длиной дуги, $\gamma(0)=o_{\alpha}, \gamma(l)=p$. Если рассмотрим $\gamma_{l}=f_{m_{l}}(\gamma)$, то они являются геодезическими на $f_{m_{l}}\left(L_{\alpha}\right)$. По условиям теоремы $\gamma_{l}(0) \rightarrow p_{0}$ при $l \rightarrow \infty$, где $p_{0}$ - некоторая точка из $M$. Тогда по теореме 2 из последовательности $\left\{\gamma_{l}(s)\right\}$ можно выделить подпоследовательность, которая поточечно сходится к некоторой геодезической $\gamma(0): \mathbb{R}^{1} \rightarrow L\left(p_{0}\right)$ слоя $L\left(p_{0}\right)$, выходящей из точки $p_{0}$ при $s=0$. Не ограничивая общности, будем считать, что сама последовательность $\left\{\gamma_{l}(s)\right\}$ сходится к $\left\{\gamma_{0}(s)\right\}$ для каждого $s \in[0 ; l]$. Отсюда следует, что $\varphi(\gamma)=\gamma_{0}$, т.е. отображение $\varphi$ изометрично переводит $L_{\alpha}$ на $L\left(p_{0}\right)$.

Теперь покажем, что $f_{m_{l}}$ сходится к $\varphi$ равномерно на каждом компакте, лежащем на слое слоения $F$. Пусть $K$ - компактное множество на слое $L$ и $\varepsilon>0$. Так как $K$ - компакт, то существует $(\varepsilon / 3)$-сеть, состоящая из конечного числа точек $\left(p_{1}, \ldots, p_{m}\right)$, где $p_{i} \in L$. Для каждой точки $p_{i}$ существует такой номер $N_{i}$, что

$$
d\left(f_{m_{l}}\left(p_{i}\right), \varphi\left(p_{i}\right)\right)<\frac{\varepsilon}{3}
$$

для произвольного $m_{l} \geqslant N_{i}$. Кроме того, для каждой точки $p \in K$ существует такое $p_{i}$, что $d_{L}\left(p, p_{i}\right)<\varepsilon / 3$. Отсюда следует, что

$$
\begin{aligned}
& d\left(f_{m_{l}}(p), \varphi(p)\right) \leqslant d\left(f_{m_{l}}(p), f_{m_{l}}\left(p_{i}\right)\right)+d\left(f_{m_{l}}\left(p_{i}\right), \varphi\left(p_{i}\right)\right)+d\left(\varphi\left(p_{i}\right), \varphi(p)\right) \leqslant \\
& \quad \leqslant d_{m_{l}}\left(f_{m_{l}}(p), f_{m_{l}}\left(p_{i}\right)\right)+d\left(f_{m_{l}}\left(p_{i}\right), \varphi\left(p_{i}\right)\right)+d_{l}\left(\varphi\left(p_{i}\right), \varphi(p)\right) \leqslant \frac{\varepsilon}{3}+\frac{\varepsilon}{3}+\frac{\varepsilon}{3}=\varepsilon
\end{aligned}
$$

для $m_{l}>N=\max _{1 \leqslant i \leqslant m}\left\{N_{i}\right\}$, где $1 \leqslant i \leqslant m$. Следовательно, получаем, что последовательность $f_{m_{l}}$ сходится к $\varphi$ в $F$-компактно-открытой топологии.

\section{СПИСОК ЛИТЕРАТУРЫ}

1. Громол Д., Клингенберг В., Мейер В. Риманова геометрия в целом. - М.: Мир, 1971.

2. Кобаяси Ш., Номидзу К. Основы дифференциальной геометрии. - М.: Наука, 1981.

3. Лукацкий А. М. Конечнопорожденность групп диффеоморфизмов// Усп. мат. наук. $-1978 .-33$, № 1 (199). - C. 219-220.

4. Лукацкий A. М. Исследование геодезического потока на бесконечномерной группе Ли с использованием оператора коприсоединенного действия// Тр. Мат. ин-ти им. В. А. Стеклова РАН. - 2009. 267. - C. 204-213.

5. Нарманов А. Я. О геометрии вполне геодезических римановых слоений// Мат. тр. -1999 . -2 , № 2 . - C. 98-106.

6. Нарманов А. Я., Саитова С. О геометрии орбит векторных полей Киллинга// Диффер. уравн. 2014. - 50, № 12. - С. 1582-1589.

7. Нарманов А. Я., Скоробогатов Д. Изометрические отображения слоений// Докл. Акад. наук Респ. Узбекистан. - 2004. - № 4. - С. 12-16.

8. Рохлин В. А., Фукс Д. Б. Начальный курс топологии. Геометрические главы. - М.: Наука, 1977.

9. Arnold $V$. Sur la geometrie differentielle des groupes de Lie de dimenzion infnite et ses applications a l'hidrodynamique des uides parfaits// Ann. Inst. Fourier. - 1966. — 16, № 1. - P. 319-361.

10. Helgason S. Differential Geometry, Lie Groups and Symmetric Spaces. - Toronto: Academic Press, 1978. 
11. Myers S., Steenrod N. The group of isometries of a Riemannian manifold// Ann. Math. - 1939. - 40, № 2. - P. 400-416.

12. Narmanov A., Sharipov A. On the group of foliation isometries// Meth. Funct. Anal. Topol. — 2009. 15, № 2. - P. 195-200.

13. Omori H. On the group of diffeomorphisms on a compact manifold// Proc. Symp. Pure Math. — 1970. 15. - P. 167-183.

14. Omori H. Groups of diffeomorphisms and thier subgroups// Trans. Am. Math. Soc - 1973. — 179. P. $85-122$.

15. Tondeur P. Foliations on Riemannian Manifolds. — New York: Springer-Verlag, 1988.

Нарманов Абдигаппар Якубович

Национальный университет Узбекистана имени Мирзо Улугбека, Ташкент, Узбекистан

E-mail: narmanov@mail.ru

Шарипов Анваржон Солиевич

Национальный университет Узбекистана имени Мирзо Улугбека, Ташкент, Узбекистан

E-mail: asharipov@inbox.ru 